\title{
ON QUASI-ORTHOGONAL POLYNOMIALS
}

\section{DAVID DICKINSON}

Let $\left\{q_{n}(x)\right\}_{n=0}^{\infty}$ be a set of polynomials, where each $q_{n}(x)$ is of degree precisely $n$. The $\left\{q_{n}(x)\right\}$ are quasi-orthogonal if there exists an interval $(a, b)$ and an associated nondecreasing function $\alpha(x)$ such that

$$
\int_{a}^{b} x^{m} q_{n}(x) d \alpha(x) \neq \begin{cases}0 & \text { for } 0 \leqq m \leqq n-2 . \\ 0 & \text { for } 0 \leqq m=n-1, \\ 0 & \text { for } 0=m=n .\end{cases}
$$

Riesz [8] and Chihara [2] have shown that a necessary and sufficient condition for the quasi-orthogonality of the $\left\{q_{n}(x)\right\}$ is that there exist nonzero constants, $\left\{a_{n}\right\}_{n=0}^{\infty}$ and $\left\{b_{n}\right\}_{n=1}^{\infty}$, such that

$$
\begin{aligned}
& q_{n}(x)=a_{n} p_{n}(x)+b_{n} p_{n-1}(x), \quad n \geqq 1, \\
& q_{0}(x)=a_{0} p_{0}(x)
\end{aligned}
$$

where the $\left\{p_{n}(x)\right\}_{n=0}^{\infty}$ are the related orthogonal polynomials. We say that two polynomial sets are related if one set is quasi-orthogonal with respect to the interval and distribution of the orthogonality of the other set. Chihara [2] gave a necessary form for a recurrence relation for quasi-orthogonal polynomials. It is the purpose of this paper to present, with an example, a recurrence relation that is both necessary and sufficient for the quasi-orthogonality of a polynomial set.

THEOREM 1. For $\left\{q_{n}(x)\right\}$ to be a set of polynomials quasi-orthogonal with respect to an interval $(a, b)$ and a distribution $d \alpha(x)$, it is necessary and sufficient that there exist a set of nonzero constants $\left\{T_{i}\right\}_{i=0}^{\infty}$ and $a$ set of polynomials $\left\{p_{n}(x)\right\}$ orthogonal with respect to $(a, b)$ and $d \alpha(x)$ such that

$$
p_{n}(x)=\sum_{i=0}^{n} T_{i} q_{i}(x),
$$$$
n \geqq 0 \text {. }
$$

NeCEssity. The quasi-orthogonality of the $\left\{q_{n}(x)\right\}$ implies the existence of a relevant interval and distribution of the quasi-orthogonality and hence the existence of a related set of orthogonal polynomials $\left\{p_{n}(x)\right\}$. Since the polynomials of each set are of degree precisely $n$, we may write

$$
p_{n}(x)=\sum_{i=0}^{n} B_{n, i} q_{i}(x), \quad n \geqq 0 .
$$

Received by the editors February 11, 1960 and, in revised form, April 18, 1960. 
Now $B_{n, 0} \neq 0$ for all $n$. For suppose there were an $m$ such that $B_{m, 0}=0$. Then multiplying the identity

$$
p_{m}(x)=\sum_{i=1}^{m} B_{m, i} q_{i}(x)
$$

successively by $d \alpha(x), x d \alpha(x), \cdots, x^{m-1} d \alpha(x)$ and integrating over $(a, b)$, we would have respectively, $B_{m, 1}=0, B_{m, 2}=0, \cdots, B_{m, m}=0$. It would then follow that $\bar{p}_{m}(x)$ would be identically zero and therefore, absurdly, not of degree $m$.

We next normalize the orthogonal polynomials of (4) by setting $p_{n}(x)=p_{n}(x) / B_{n, 0}$. The identity (4) then takes the form

$$
p_{n}(x)=\sum_{i=0}^{n} T_{n, i} q_{i}(x), \quad n \geqq 0,
$$

with

$$
T_{n, 0}=T_{0,0} \neq 0 \quad \text { for } n \geqq 0 .
$$

This implies that

$$
p_{n}(x)-p_{n-1}(x)=T_{n, n} q_{n}(x)+\sum_{i=1}^{n-1}\left(T_{n, i}-T_{n-1, i}\right) q_{i}(x), \quad n \geqq 1,
$$

where, here as well as later, we take $\sum_{i=1}^{0} a_{i}$ to be zero. Multiplying successively by $d \alpha(x), x d \alpha(x), \cdots, x^{n-2} d \alpha(x)$, and integrating over $(a, b)$, we see that

$$
\begin{gathered}
T_{n, 1}-T_{n-1,1}=0 \\
T_{n, 2}-T_{n-1,2}=0 \\
\cdot \cdot \cdot \cdot \cdot \\
T_{n, n-1}-T_{n-1, n-1}=0 .
\end{gathered}
$$

Since $n$ may be taken arbitrarily large, we may thus set $T_{i}=T_{n, i}$ for all $n$ and $i$ for which $T_{n, i}$ is defined. Hence we have

$$
p_{n}(x)=\sum_{i=0}^{n} T_{i} q_{i}(x), \quad n \geqq 0 .
$$

If one of the $\left\{T_{i}\right\}$, say $T_{m}$, were zero, then the absurdity that $p_{m}(x)$ is of degree less than $m$ would follow.

SUfFICIENCY. We prove the sufficiency by noting that

$$
p_{n}(x)=\sum_{i=0}^{n} T_{i} q_{i}(x), \quad n \geqq 0,
$$

implies that 


$$
\begin{aligned}
p_{n}(x)-p_{n-1}(x) & =T_{n} q_{n}(x), & n \geqq 1, \\
p_{0}(x) & =T_{0} q_{0}(x), &
\end{aligned}
$$

expressions equivalent to (2). Q.E.D.

THEOREм 2. A necessary and sufficient condition that the set $\left\{q_{n}(x)\right\}_{n=0}^{\infty}$, where each $q_{n}(x)$ is a polynomial of degree precisely $n$, be quasi-orthogonal is that it satisfy

(5) $x q_{n}(x)=A_{n} q_{n+1}(x)+B_{n} q_{n}(x)+C_{n} q_{n-1}(x)+D_{n} \sum_{i=0}^{n-2} T_{i} q_{i}(x)$ for all $n$ where $T_{i} \neq 0$,

$$
\begin{aligned}
& \frac{A_{n-3} T_{n-3}}{T_{n-2}}-\frac{A_{n-2} T_{n-2}}{T_{n-1}}+B_{n-2}-B_{n-1} \\
& +\frac{C_{n-1} T_{n-1}}{T_{n-2}}-\frac{C_{n} T_{n}}{T_{n-1}}+D_{n} T_{n}=0 \quad \text { far all } n
\end{aligned}
$$

and

$$
\frac{A_{n-1} T_{n-1}}{T_{n}}\left(\frac{C_{n+1} T_{n+1}}{T_{n}}+D_{n+1} T_{n+1}\right)>0 \quad \text { for } n \geqq 1,
$$

and where any terms involving $A_{n}, B_{n}, C_{n+1}, D_{n+2}$, or $q_{n}(x)$ with negative $n$ are taken to be zero.

NeCEssity. The existence of the quasi-orthogonal $\left\{q_{n}(x)\right\}$ implies the existence of a set of related orthogonal polynomials $\left\{p_{n}(x)\right\}$ such that

$$
p_{n}(x)=\sum_{i=0}^{n} T_{i} q_{i}(x)
$$

Since the $n$th polynomial of each set is of degree precisely $n$, there exist coefficients such that

(9) $x q_{n}(x)=A_{n} q_{n+1}(x)+B_{n} q_{n}(x)+C_{n} q_{n-1}(x)+\sum_{i=0}^{n-2} A_{n, i} p_{i}(x)$ for $n \geqq 2$.

If we multiply (9) successively by $d \alpha(x), x d \alpha(x), \cdots, x^{n-3} d \alpha(x)$ and integrate over $(a, b)$, we see that, respectively, $A_{n, 0}=0, A_{n ; 1}=0, \cdots$, $A_{n, n-3}=0$. Hence we have

$$
x q_{n}(x)=A_{n} q_{n+1}(x)+B_{n} q_{n}(x)+C_{n} q_{n-1}(x)+D_{n} p_{n-2}(x), \quad n \geqq 2,
$$

or 
$x q_{n}(x)=A_{n} q_{n+1}(x)+B_{n} q_{n}(x)+C_{n} q_{n-1}(x)+D_{n} \sum_{i=0}^{n-2} T_{i} q_{i}(x), \quad n \geqq 2$.

For the special cases $n<2$, the expansion of $x q_{n}(x)$ according to (5) can be verified in the light of the coefficient conventions described in the theorem. From (8) we may write, where $p_{n}(x)=0$ for $n<0$,

$$
T_{n} q_{n}(x)=p_{n}(x)-p_{n-1}(x)
$$

for all $n$

and hence we see that (5) may be written

$$
\begin{array}{r}
x\left(p_{n}(x)-p_{n-1}(x)\right)=\frac{A_{n} T_{n}}{T_{n+1}}\left(p_{n+1}(x)-p_{n}(x)\right)+B_{n}\left(p_{n}(x)-p_{n-1}(x)\right) \\
+\frac{C_{n} T_{n}}{T_{n-1}}\left(p_{n-1}(x)-p_{n-2}(x)\right)+D_{n} T_{n} p_{n-2}(x) \quad \text { for all } n .
\end{array}
$$

The possible appearance of $T_{i}$ 's with negative $i$ is purely formal. Any such $T_{i}$ may be given arbitrary nonzero values and, from the coefficient conventions described in the theorem, the terms involving such $T_{i}$ are zero. We may rewrite (10) as

$$
\begin{aligned}
x\left(p_{n}(x)-p_{n-1}(x)\right) & =\frac{A_{n} T_{n}}{T_{n+1}} p_{n+1}(x)+\left(B_{n}-\frac{A_{n} T_{n}}{T_{n+1}}\right) p_{n}(x) \\
+ & \left(\frac{C_{n} T_{n}}{T_{n-1}}-B_{n}\right) p_{n-1}(x)+\left(D_{n} T_{n}-\frac{C_{n} T_{n}}{T_{n-1}}\right) p_{n-2}(x)
\end{aligned}
$$

or

$\left.\begin{array}{r}x p_{n}(x) \\ x p_{n-1}(x)\end{array}\right\}$

$$
\begin{aligned}
&=\left\{\begin{array}{l}
\frac{A_{n} T_{n}}{T_{n+1}} p_{n+1}(x)+\left(\frac{A_{n-1} T_{n-1}}{T_{n}}-\frac{A_{n} T_{n}}{T_{n+1}}+B_{n}\right) p_{n}(x) \\
-\frac{A_{n-1} T_{n-1}}{T_{n}} p_{n}(x)-\left(\frac{A_{n-2} T_{n-2}}{T_{n-1}}-\frac{A_{n-1} T_{n-1}}{T_{n}}+B_{n-1}\right) p_{n-1}(x)
\end{array}\right. \\
&\left\{\begin{array}{r}
+\left(\frac{A_{n-2} T_{n-2}}{T_{n-1}}-\frac{A_{n-1} T_{n-1}}{T_{n}}+B_{n-1}-B_{n}+\frac{C_{n} T_{n}}{T_{n-1}}\right) p_{n-1}(x) \\
-\left(\frac{A_{n-3} T_{n-3}}{T_{n-2}}-\frac{A_{n-2} T_{n-2}}{T_{n-1}}+B_{n-2}-B_{n-1}+\frac{C_{n-1} T_{n-1}}{T_{n-2}}\right) p_{n-2}(x)
\end{array}\right. \\
&+\left(\frac{A_{n-3} T_{n-3}}{T_{n-2}}-\frac{A_{n-2} T_{n-2}}{T_{n-1}}+B_{n-2}-B_{n-1}+\frac{C_{n-1} T_{n-1}}{T_{n-2}}\right. \\
&\left.-\frac{C_{n} T_{n}}{T_{n-1}}+D_{n} T_{n}\right) p_{n-2}(x) \quad \text { for all } n .
\end{aligned}
$$


At this point we need a lemma.

Lemma 1. A necessary and sufficient condition that the polynomials $\left\{R_{n}(x)\right\}_{n=-1}^{\infty}$ (where $R_{-1}(x)=0$ and every other $R_{n}(x)$ is of degree precisely $n$ ) be orthogonal is that the $R_{n}(x)$ satisfy

$$
x R_{n}(x)=\alpha_{n} R_{n+1}(x)+\beta_{n} R_{n}(x)+\gamma_{n} R_{n-1}(x) \quad \text { for } n \geqq 0
$$

where

$$
\alpha_{n-1} \gamma_{n}>0 \quad \text { for } n \geqq 1 .
$$

Proof. Since $\alpha_{n} \neq 0$ for $n \geqq 0$ implies and is implied by the fact that polynomials of degree precisely $n$ satisfy the recurrence relation, (12) may be written

$$
\begin{aligned}
x\left[R_{n}(x) \prod_{i=0}^{n-1} \alpha_{i}\right]= & {\left[R_{n+1}(x) \prod_{i=0}^{n} \alpha_{i}\right]+\beta_{n}\left[R_{n}(x) \prod_{i=0}^{n-1} \alpha_{i}\right] } \\
& +\gamma_{n} \alpha_{n-1}\left[R_{n-1}(x) \prod_{i=0}^{n-2} \alpha_{i}\right] \quad \text { for } n \geqq 0
\end{aligned}
$$

where an empty product is to be interpreted as unity. But according to a theorem of Favard [6] or Shohat [9], the polonomials $\left\{R_{n}(x) \prod_{i=0}^{n-1} \alpha_{i}\right\}$ of (14) (and hence the polynomials $\left\{R^{n}(x)\right\}$ ) are orthogonal if and only if

$$
\alpha_{n-1} \gamma_{n}>0
$$

for $n \geqq 1$.

Now the polynomials $\left\{p_{n}(x)\right\}$ of (11) are orthogonal. Hence they must obey a relation of the form (12). Comparing (11) and (12), we see that we must have $\alpha_{n}=A_{n} T_{n} / T_{n+1}, \alpha_{n-1}=A_{n-1} T_{n-1} / T_{n}, \beta_{n}$ $=\left(A_{n-1} T_{n-1} / T_{n}\right)-\left(A_{n} T_{n} / T_{n+1}\right)+B_{n}$, etc. That is, the polynomials of (11) satisfy

$$
\begin{aligned}
x p_{n}(x)= & \frac{A_{n} T_{n}}{T_{n+1}} p_{n+1}(x)+\left(\frac{A_{n-1} T_{n-1}}{T_{n}}-\frac{A_{n} T_{n}}{T_{n+1}}+B_{n}\right) p_{n}(x) \\
& +\left(\frac{A_{n-2} T_{n-2}}{T_{n-1}}-\frac{A_{n-1} T_{n-1}}{T_{n}}+B_{n-1}-B_{n}+\frac{C_{n} T_{n}}{T_{n-1}}\right) p_{n-1}(x) \\
& \text { for } n \geqq 0
\end{aligned}
$$

where

$$
\begin{aligned}
\frac{A_{n-3} T_{n-3}}{T_{n-2}}-\frac{A_{n-2} T_{n-2}}{T_{n-1}}+B_{n-2} & -B_{n-1} \\
& +\frac{C_{n-1} T_{n-1}}{T_{n-2}}-\frac{C_{n} T_{n}}{T_{n-1}}+D_{n} T_{n}=0
\end{aligned}
$$


for $n \geqq 1$ and, because of the conventions, also for $n<1$. Condition (13) applied to (15) becomes

$$
\begin{aligned}
\frac{A_{n-1} T_{n-1}}{T_{n}}\left(\frac{A_{n-2} T_{n-2}}{T_{n-1}}-\frac{A_{n-1} T_{n-1}}{T_{n}}+B_{n-1}-B_{n}+\frac{C_{n} T_{n}}{T_{n-1}}\right) & >0, \\
n & \geqq 1,
\end{aligned}
$$

or, using (6) to simplify the second factor,

$$
\frac{A_{n-1} T_{n-1}}{T_{n}}\left(\frac{C_{n+1} T_{n+1}}{T_{n}}+D_{n+1} T_{n+1}\right)>0 \quad \text { for } n \geqq 1 .
$$

Sufficiency. Suppose that we have a polynomial set $\left\{q_{n}(x)\right\}$ that satisfies (5) with the associated conditions on the coefficients of (5) as given in the theorem. Let us define a polynomial set $\left\{p_{n}(x)\right\}$ by the relation

$$
p_{n}(x)=\sum_{i=0}^{n} T_{i} q_{i}(x) \quad \text { or } \quad T_{n} q_{n}(x)=p_{n}(x)-p_{n-1}(x)
$$

where $p_{n}(x)=0$ for $n<0$. These polynomials will then satisfy (11). Condition (6) placed on (11) implies that (11) may be written in the form

$$
\begin{aligned}
x\left(p_{n}(x)-p_{n-1}(x)\right)= & \alpha_{n} p_{n+1}(x)+\left(\beta_{n}-\alpha_{n-1}\right) p_{n}(x) \\
& +\left(\gamma_{n}-\beta_{n-1}\right) p_{n-1}(x)-\gamma_{n-1} p_{n-2}(x) \text { for all } n .
\end{aligned}
$$

If we take (18) and the expressions formed from it by shifting $n$ to $n-1$ a sufficient number of times to obtain an expression where all the terms are zero, and then add all these expressions together, we get

$$
x p_{n}(x)=\alpha_{n} p_{n+1}(x)+\beta_{n} p_{n}(x)+\gamma_{n} p_{n-1}(x)
$$

for all $n$.

Since (7) combined with (6) states that $\alpha_{n-1} \gamma_{n}>0$ for $n \geqq 1$, our lemma shows that the $\left\{p_{n}(x)\right\}$ are orthogonal and hence that the $\left\{q_{n}(x)\right\}$ are quasi-orthogonal. Q.E.D.

If we have a set of quasi-orthogonal polynomials for which the coefficient $D_{n}$ in (5) never vanishes, we may multiply (5) by $D_{n}^{-1}=\alpha_{n}$, shift the index from $n$ to $n-1$, subtract the two relations and so obtain a recurrence formula of the form

$$
\begin{array}{r}
x\left(\alpha_{n} q_{n}(x)-\alpha_{n-1} q_{n-1}(x)\right) \\
\quad=\beta_{n} q_{n+1}(x)+\gamma_{n} q_{n}(x)+\delta_{n} q_{n-1}(x)+\epsilon_{n} q_{n-2}(x) .
\end{array}
$$

Many polynomial sets that have appeared in the literature (e.g. 
Rice's $H_{n}(\zeta, p, v)$ [7]; Bateman's $Z_{n}(x)$ [1]; and Sister Celine's $f_{n}(a ;-; x)$ and $\left.f_{n}(-;-; x)[5 ; 4]\right)$ possess a recurrence relation of this form. Hence we are led to ask if some of these polynomials are quasi-orthogonal.

Let us assume that we have a polynomial set $\left\{q_{n}(x)\right\}$, each $q_{n}(x)$ being of degree precisely $n$ for $n \geqq 0$ and $q_{n}(x)=0$ for $n<0$, that satisfies (20) for all $n$. Further, let us assume that $\alpha_{n} \neq 0$ for all $n$. Since $q_{n}(x)$ for non-negative $n$ is of degree precisely $n$, there exist coefficients $A_{n, k}$ such that

$$
x \alpha_{n} q_{n}(x)=\sum_{k=0}^{n+1} A_{n, k} q_{k}(x), \quad n \geqq 0 .
$$

After substituting the sum for $x \alpha_{n} q_{n}(x)$ and $x \alpha_{n-1} q_{n-1}(x)$ in (20) we obtain the identities

$$
\begin{aligned}
& A_{n, n+1}=\beta_{n} \\
& A_{n, n}-A_{n-1, n}=\gamma_{n} \\
& A_{n, n-1}-A_{n-1, n-1}=\delta_{n} \quad \text { for all } n \text { and } k=0,1, \cdots, n-3 . \\
& A_{n, n-2}-A_{n-1, n-2}=\epsilon_{n} \\
& A_{n, k}-A_{n-1, k}=0
\end{aligned}
$$

That is,

$$
\begin{aligned}
x \alpha_{n} q_{n}(x)= & \beta_{n} q_{n+1}(x) \\
& +\left(\beta_{n-1}+\gamma_{n}\right) q_{n}(x) \\
& +\left(\beta_{n-2}+\gamma_{n-1}+\delta_{n}\right) q_{n-1}(x) \\
& +\sum_{k=0}^{n-2}\left(\beta_{k-1}+\gamma_{k}+\delta_{k+1}+\epsilon_{k+2}\right) q_{k}(x)
\end{aligned}
$$

for $n \geqq 0$ where the sum is taken to be zero if the lower summation limit exceeds the upper summation limit. This relation (23) can easily be written in the form of (5) and the side conditions on (5) can then be used to obtain the conditions on the coefficients of (20) that will assure us that the $q_{n}(x)$ of (20) and (23) are quasi-orthogonal. Such conditions on the coefficients of (20) can be obtained by a straightforward procedure but the expressions in this general case are too complicated to profitably reproduce here.

Let us now specialize our polynomials to Sister Celine's $f_{n}(a ;-; x)$ $=f_{n}(a, x)$. The $f_{n}(a, x)$ are generalized hypergeometric polynomials.

$$
f_{n}(a, x)={ }_{3} F_{2}(-n, n+1, a ; 1 / 2,1 ; x)=\sum_{k=0}^{\infty} \frac{(-n)_{k}(n+1)_{k}(a)_{k} x^{k}}{(1 / 2)_{k}(1)_{k} k !}
$$


They have, from [5], the recurrence relation

$$
\begin{aligned}
& n f_{n}(a, x)-[(3 n-2)-4(n-1+a) x] f_{n-1}(a, x) \\
& \quad+[(3 n-4)-4(n-1-a) x] f_{n-2}(a, x)-(n-2) f_{n-3}(a, x)=0
\end{aligned}
$$

for all $n$.

This recurrence relation may be written in the form (20):

$$
\begin{aligned}
x & \frac{(1+a)_{n}}{(1-a)_{n}} f_{n}(a, x)-x \frac{(1+a)_{n-1}}{(1-a)_{n-1}} f_{n-1}(a, x) \\
= & -\frac{(n+1)(1+a)_{n-1}}{4(1-a)_{n}} f_{n+1}(a, x)+\frac{(3 n+1)(1+a)_{n-1}}{4(1-a)_{n}} f_{n}(a, x) \\
& \quad-\frac{(3 n-1)(1+a)_{n-1}}{4(1-a)_{n}} f_{n-1}(a, x)+\frac{(n-1)(1+a)_{n-1}}{4(1-a)_{n}} f_{n-2}(a, x)
\end{aligned}
$$

for all $n$.

This recurrence relation implies that the $f_{n}(a, x)$ satisfy a relation of the form of (23). If we form this relation and divide both sides by $\alpha_{n}$, we obtain a relation of the form (5):

$$
\begin{aligned}
x f_{n}(a, x)= & -\frac{(n+1)}{4(n+a)} f_{n+1}(a, x) \\
& +\left[\frac{(3 n+1)}{4(n+a)}-\frac{(n)(n-a)}{4(n+a)(n+a-1)}\right] f_{n}(a, x) \\
& +\left[\frac{-(3 n-1)}{4(n+a)}+\frac{(3 n-2)(n-a)}{4(n+a)(n+a-1)}\right. \\
& \left.\quad-\frac{(n-1)(n-a)(n-a-1)}{4(n+a)(n+a-1)(n+a-2)}\right] f_{n-1}(a, x) \\
& +\frac{(2 a-1)(a-1)^{2}(3-a)_{n-2}}{2(a-1)_{n+2}} \sum_{k=0}^{n-2} \frac{(2 k+1)(a-1)_{k}}{(3-a)_{k}} f_{k}(a, x) .
\end{aligned}
$$

We observe that if $a$ is $1 / 2$ or 1 , (25) degenerates into an orthogonal type recurrence relation. From [5], the polynomials thus defined are specialized asymmetric Jacobi polynomials. Explicitly, we have

$$
\begin{aligned}
f_{n}(1 / 2, x) & =P_{n}(1-2 x), \\
f_{n}(1, x) & =\frac{n !}{(1 / 2)_{n}} P_{n}^{(-1 / 2,1 / 2)}(1-2 x) .
\end{aligned}
$$

We seek those polynomials that satisfy (25) that are quasi-orthog- 
onal. That is, we seek those values of the parameter $a$ for which the conditions (6) and (7) are met. After a straightforward but tedious calculation, the left member of (6) becomes for the special polynomials of (25),

$$
\frac{(n-1)(a-2)^{2}(2 a-3)}{(n+a-4)(n+a-3)(n-a+1)(n-a+2)} .
$$

This expression is zero when $a=2$ or $3 / 2$. When $a=2$ or $3 / 2$, the inequality (7) is also satisfied. Therefore the polynomial sets $\left\{f_{n}(2, x)\right\}$ and $\left\{f_{n}(3 / 2, x)\right\}$ are quasi-orthogonal.

Let us first consider the polynomials $\left\{f_{n}(2, x)\right\}$. Setting $a=2$ in (25) and putting the resulting coefficients $A_{n}, B_{n}, C_{n}, D_{n}$, and $T_{n}$ in (15), we obtain the recurrence relation for the orthogonal polynomials $\left\{p_{n}(2, x)\right\}$ that are related to the $\left\{f_{n}(2, x)\right\}$ :

$$
\begin{aligned}
x p_{n}(2, x)= & \frac{-(2 n+1)(n+1)}{4(n+2)(2 n+3)} p_{n+1}(2, x) \\
& +\frac{8 n^{2}+16 n+2}{4(2 n+3)(2 n+1)} p_{n}(2, x) \\
& -\frac{(2 n+3)(n+1)}{4(n)(2 n+1)} p_{n-1}(2, x) .
\end{aligned}
$$

Because asymmetric Jacobi polynomials have turned up in the derivation of this expression, one suspects that the $p_{n}(2, x)$ are certain specialized asymmetric Jacobi polynomials. Changing the variable $x$ to $(1-z) / 2$ in $(27)$ and rewriting the expression with an eye on the recurrence relation of the Jacobi polynomials (Erdélyi $[3,10.8 .11]$ ), we obtain

$$
\begin{aligned}
2(n+1)(n+2)(2 n+1)\left\{\frac{(1 / 2)_{n+1} p_{n+1}(2,(1-z) / 2)}{(n+2) !(n+2)}\right\} \\
=(2 n+2)[z(2 n+1)(2 n+3)-2]\left\{\frac{(1 / 2)_{n} p_{n}(2,(1-z) / 2)}{(n+1) !(n+1)}\right\} \\
\quad-2(n-1 / 2)(n+3 / 2)(2 n+3)\left\{\frac{(1 / 2)_{n-1} p_{n-1}(2,(1-z) / 2)}{n !(n)}\right\} .
\end{aligned}
$$

It is now obvious that we are indeed working with an asymmetric Jacobi polynomial and that $\alpha=-1 / 2$ and $\beta=3 / 2$. Hence we have

$$
\frac{(1 / 2)_{n} p_{n}(2,(1-z) / 2)}{(n+1) !(n+1)}=P_{n}^{(-1 / 2,3 / 2)}(z)
$$


or

$$
p_{n}(2, x)=\frac{(n+1) !(n+1)}{(1 / 2)_{n}} P_{n}^{(-1 / 2,3 / 2)}(1-2 x) .
$$

Thus we have, from

$$
q_{n}(x)=T_{n}^{-1}\left(p_{n}(x)-p_{n-1}(x)\right)
$$

that

$$
\begin{gathered}
f_{n}(2, x)=\frac{1}{(2 n+1)}\left\{\frac{(n+1) !(n+1)}{(1 / 2)_{n}} P_{n}^{(-1 / 2,3 / 2)}(1-2 x)\right. \\
\left.\quad-\frac{n !(n)}{(1 / 2)_{n-1}} P_{n-1}^{(-1 / 2,3 / 2)}(1-2 x)\right\}
\end{gathered}
$$

and the polynomials $\left\{f_{n}(2, x)\right\}$ are quasi-orthogonal over the interval $(0,1)$ with respect to the weight function $x^{-1 / 2}(1-x)^{3 / 2}$.

Similarly, it can be shown that

$$
f_{n}(3 / 2, x)=(n+1) P_{n}^{(0,1)}(1-2 x)-n P_{n-1}^{(0,1)}(1-2 x)
$$

and therefore the polynomials $\left\{f_{n}(3 / 2, x)\right\}$ are quasi-orthogonal over the interval $(0,1)$ with respect to the weight function $(1-x)$.

\section{BIBLIOGRAPHY}

1. H. Bateman, Two systems of polynomials for the solution of Laplace's integral equation, Duke Math. J. vol. 2 (1936) pp. 569-577.

2. T. S. Chihara, On quasi-orthogonal polynomials, Proc. Amer. Math. Soc. vol. 8 (1957) pp. 765-767.

3. A. Erdélyi et al., Higher transcendental functions, New York, McGraw-Hill, 1953.

4. M. C. Fasenmyer, Some generalized hypergeometric polynomials, Bull. Amer. Math. Soc. vol. 65 (1949) pp. 806-812.

5. - A note on pure recurrence relations, Amer. Math. Monthly vol. 56 (1949) pp. 14-17.

6. J. Favard, Sur les polynomes de Tchebicheff, C. R. Acad. Sci. Paris vol. 200 (1935) pp. 2052-2053.

7. S. O. Rice, Some properties of ${ }_{3} F_{2}(-n, n+1, \zeta ; 1, p ; v)$, Duke Math. J. vol. 6 (1940) pp. 108-119.

8. M. Riesz, Sur le problème des moments. Troisième note, Arkiv för Matematik, Astronomi och Fysik, vol. 17 (1923).

9. J. Shohat, The relation of the classical orthogonal polynomials to the polynomials of Appell, Amer. J. Math. vol. 58 (1936) pp. 453-464.

UNIVERSITY OF MASSACHUSETTS 